\title{
钉催化的直接不对称还原胺化合成手性二芳基甲胺与大位阻胺
}

\author{
黄海洲常明欣* \\ (西北农林科技大学化学与药学院 陕西杨凌 712100)
}

\section{Ruthenium-Catalyzed Direct Asymmetric Reductive Amination for the Synthesis of Chiral Diarylmethylamines and Sterically Hindered Amines}

\author{
Huang, Haizhou Chang, Mingxin* \\ (College of Chemistry \& Pharmacy, Northwest A\&F University, Yangling, Shaanxi 712100)
}

手性二芳基甲胺和大位阻胺是天然产物、药物和生 物活性化合物的重要砌块 ${ }^{[1]}$, 也是手性配体的合成和催 化剂设计中的关键要素 ${ }^{[2]}$. 其主要合成方法包括外消旋 体的拆分，芳基金属或芳基硼试剂对亚胺的不对称加 成 ${ }^{[3]}$ 和亚胺的不对称氢化 ${ }^{[4]}$ 等. 对于氮上没有保护基团 的手性二芳基甲胺的合成, 因为氮对过渡金属的强配位 能力而导致的抑制作用 ${ }^{[5,6]}$, 相应成功的不对称催化报 道非常少见. 汤文军团队 ${ }^{[7]}$ 利用大位阻的 Wingphos 实 现了含嗍化合物对非保护的芳基三氟甲基亚胺的不对 称加成(Scheme 1a); Zhang 课题组 ${ }^{[8]}$ 使用 Ir/N-Me $N$-Bn Monophos 催化剂完成了非保护的二芳基亚胺的不对称 氢化(Scheme 1b).

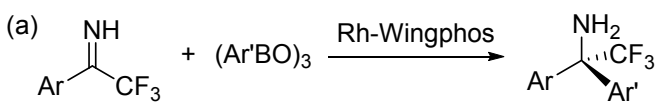

(b)<smiles>N=C([Al])[Al]</smiles>

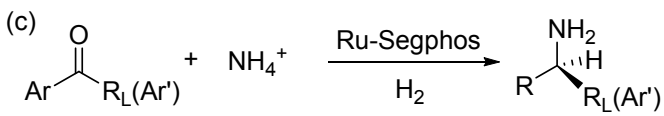

图式 1 氮上无保护的二芳基甲胺的合成方法 Scheme 1 Synthesis of $N$-unprotected chiral diaryl methamine

最近，南方科技大学张绪穆和殷勤团队 ${ }^{[9]}$ 以铵盐为 胺源, 氢气作为还原剂, Ru-Segphos 络合物作为手性催 化剂, 成功实现了二芳基酮和大位阻芳香酮的不对称还 原胺化反应, 以制备手性二芳基甲胺和大位阻芳香胺 (Scheme 1c). 作者通过在酮底物中引入邻位羟基基团以
活化羰基, 加速亚胺生成, 其与亚胺形成分子内氢键以 稳定亚胺中间体; 同时, 羊基上的氢、路易斯酸钎酸四 异丙酯 $\left(\mathrm{Ti}(\mathrm{O} i-\mathrm{Pr})_{4}\right.$ 中的钛会与氮原子形成配位键，促使 单一 $E$ 构型的亚胺生成, 从而解决二芳基酮或大位阻酮 在不对称还原胺化(ARA)反应中亚胺的生成和对映选择 性控制的难题(Scheme 2).

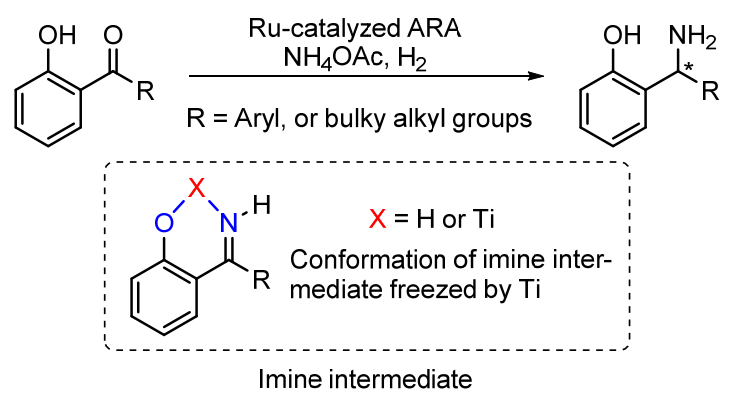

图式 2 底物引入邻羟基与亚胺形成氢键或配位键 Scheme 2 ortho-Hydroxy-substituted substrate to form hydrogen bonds or coordination bonds with imines

作者通过尝试各种酸添加剂, 最终确定以路易斯酸 $\mathrm{Ti}(\mathrm{O} i-\mathrm{Pr})_{4}$ 为添加剂, 甲醇 $(\mathrm{MeOH})$ 为溶剂, 手性配体采 用轴手性双膦配体 $(S)$-SegPhos. 该反应具有较好的底物 普适性, 在邻羟基苯基芳基酮底物进行扩展的研究中 (Scheme 3), 产物的对映选择性不受芳基取代供电子性 质 $\left(\mathrm{Me} 、 \mathrm{MeO}\right.$ 等)或者吸电子性质 $\left(\mathrm{F} 、 \mathrm{Cl} 、 \mathrm{Br} 、 \mathrm{CF}_{3}\right.$ 等) 的影响, 杂芳基底物也能与反应体系兼容; 产率方面则 受取代基电子效应的影响, 普遍来说具有吸电子取代基 产物的产率低于具有供电子取代基产物的产率. 大位阻

* Corresponding author. E-mail: mxchang@nwsuaf.edu.cn. Published online May 9, 2020. 
单芳基酮的底物扩展时，如含苄基、2-异丁基、异丙基、 环己基、叔丁基和金刚烷基的底物均能获得相应的氨基 苄基酚产物. 此外，该反应在不对称控制方面表现十分 突出，可区分两个均含邻位取代基的芳基.

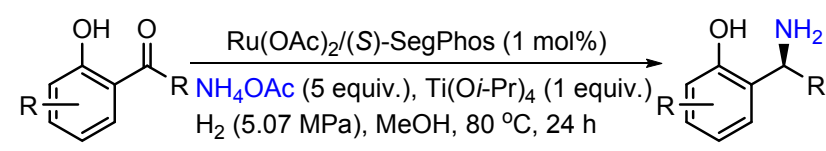

Diarylmethylamines

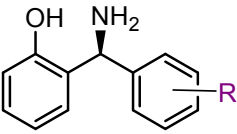

$\mathrm{R}$ at para position, 6 examples: $66 \% \sim 94 \%$ yield $93 \% \sim 99 \%$ ee

$\mathrm{R}$ at meta position, 6 examples: $70 \% \sim 97 \%$ yield $95 \% \sim 99 \%$ ee

$\mathrm{R}$ at ortho position, 3 examples: $76 \% \sim 89 \%$ yield $97 \% \sim 99 \%$ ee<smiles>N[C@@H](Br)c1ccccc1O</smiles>

$\mathrm{Ar}=$ other aromatic substituents 5 examples: $40 \% \sim 72 \%$ yield

$97 \% \sim 99 \%$ ee

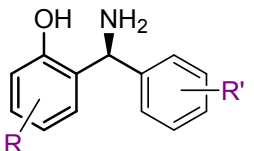

3 examples, $68 \% \sim 88 \%$ yield $94 \% \sim 99 \%$ ee
Sterically hindered amines<smiles>[R]C(N)c1ccccc1O</smiles>

6 examples, $82 \% \sim 94 \%$ yield, $98 \% \sim 99 \%$ ee

图式 3 底物范围

Scheme 3 Substrate scope

为进一步证明该反应的实用性, 作者也对产物进行 了相关的修饰(Scheme 4), 如产物中羟基的去除, 脱羟 基后的产物 2 可作为合成左西替利嗪(levocetirizine)的 关键手性中间体. 作为多种生物活性分子和天然生物碱 中的结构单元的 NH 异吲哚啉酮 4, 可通过化合物 3 经 羰基化、分子内酰胺化、 $N$-Boc 脱保护三步反应获得. 作 者也将产物应用于手性配体的合成, 由化合物 5 与丙二 酰亚胺二乙酯二盐酸盐缩合, 制备了一种新型的手性苯 并恶嗪骨架配体 6.

总之, 张绪穆和殷勤团队通过在底物上引入邻羟基 基团来形成氢键或配位键, 成功实现了邻着基取代的二 芳基和大位阻酮的直接不对称胺化还原反应. 此外, 克 级实验、手性药物左西替利嗪中间体、异吲哚啉酮化合 物以及新型苯并恶嗪配体的合成, 进一步证明了该方法 的实用性. 为手性二芳基甲胺和大位阻胺的高效合成提 (a)

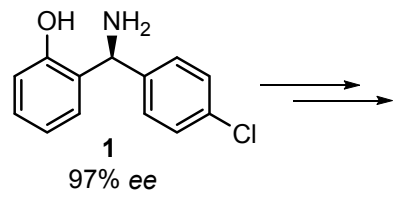<smiles>N[C@H](c1ccccc1)c1ccc(Cl)cc1</smiles>

$67 \%$ yield for 3 step $96 \%$ ee

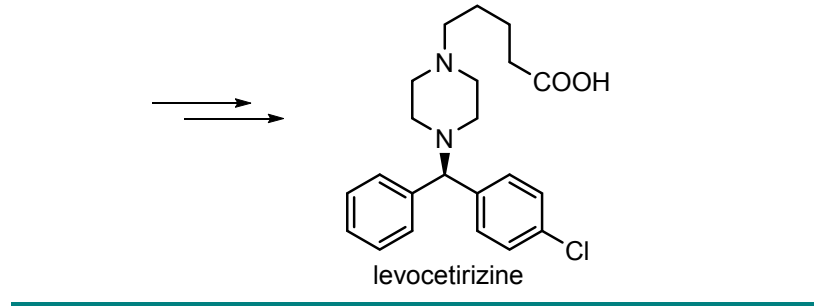

(b) $98 \%$ ee

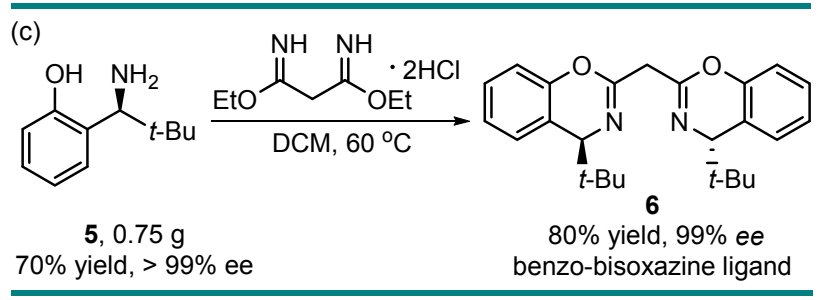

图式 4 该反应的在药物和配体合成中应用

Scheme 4 Application of the reaction in drugs and ligands synthesis

供了新途径.

\section{References}

[1] Afanasyev, O. I.; Kuchuk, E.; Usanov, D. L.; Chusov, D. Chem. Rev. 2019, 119, 11857.

[2] Zhang, Z.-M.; Chen, P.; Li, W.; Niu, Y.; Zhao, X.-L.; Zhang, J. Angew. Chem., Int. Ed. 2014, 53, 4350.

[3] Chen, D.; Xu, M.-H. Chin. J. Org. Chem. 2017, 37, 1589 (in Chinese). (陈雕，徐明华，有机化学, 2017, 37, 1589.)

[4] Xie, J.-H.; Zhu, S.-F.; Zhou, Q.-L. Chem. Rev. 2011, 111, 1713.

[5] Nugent, T. C. Chiral Amine Synthesis: Methods, Developments and Applications, Wiley-VCH, Weinheim, 2010.

[6] Li, W.; Zhang, X. In Topics in Current Chemistry, Vol. 343, Springer, Berlin, 2014.

[7] Zhu, J.; Huang, L.; Dong, W.; Li, N.; Yu, X.; Deng, W.; Tang, W. Angew. Chem., Int. Ed. 2019, 58, 16119.

[8] Hou, G.; Tao, R.; Sun, Y.; Zhang, X.; Gosselin, F. J. Am. Chem. Soc. 2010, 132, 2124

[9] Hu, L.; Zhang, Y.; Zhang, Q.; Yin, Q.; Zhang, X. Angew. Chem., Int. Ed. 2020, 59, 5321. 